Pacific

Journal of

Mathematics

\title{
ON THE LOCAL NIRENBERG PROBLEM FOR $\sigma_{k}$-TYPE CURVATURES
}

PhilipPe Delanoë 


\title{
ON THE LOCAL NIRENBERG PROBLEM FOR $\sigma_{k}$-TYPE CURVATURES
}

\author{
PhilipPe DELANOË
}

\begin{abstract}
This note is about the Nirenberg problem for a class of second-order fully nonlinear scalar curvature operators, namely those that are nondegenerate symmetric functions of the eigenvalues of the Schouten tensor. Near the standard metric in its conformal class, we prove the nonconstrainability of their local image, local existence à la Fredholm and local solvability under a symmetry assumption à la Moser. We include a remark on the KazdanWarner identities for the $\sigma_{k}$-curvatures.
\end{abstract}

\section{Introduction}

In this note we continue the study of the local Nirenberg problem begun in [Delanoë 2003; Delanoë and Robert 2007], by dealing with nondegenerate symmetric functions of the eigenvalues of the Schouten tensor, such as the so-called $\sigma_{k}$-curvatures [Sheng et al. 2007]. Relying on [Delanoë 2003, Section 4.1], we provide for such curvature operators quick proofs of a "no-constraint" theorem analogous to [Delanoë 2003, Theorem 10] and [Delanoë and Robert 2007, Theorem 2], and of a related local existence result.

Our no-constraint theorem stands in contrast with the identities of KazdanWarner type [1974] satisfied by the graphs of the conformal $\sigma_{k}$-curvatures operators when $k \leq 2$ or on locally conformally flat manifolds [Viaclovsky 2000; Han 2006]. These identities follow from [Delanoë and Robert 2007, Theorem 3] since, on the one hand, the Schouten tensor is natural [Stredder 1975], and hence so are the $\sigma_{k}$-curvatures; on the other hand, setting $F[u]=\sigma_{k}\left[\lambda\left(A_{u}\right)\right]$ (see notations below), the linearized operator $d F[u]$ is self-adjoint with respect to the conformal metric $g_{u}$, by [Sheng et al. 2007, Section 2.4].

MSC2000: primary 35J60, 53C21; secondary 58J60.

Keywords: Nirenberg problem, Schouten tensor, fully nonlinear scalar curvature, Kazdan-Warner identities, local image, nonconstrainability, local existence, symmetry.

The author is supported by the CNRS. 


\section{Notations and statement of results}

Set $A=A(g)$ for the Schouten tensor of a Riemannian metric $g$ on a manifold of dimension $n>2$. It is given by

$$
A=\frac{1}{n-2}\left(\text { Ric }-\frac{\text { Scal }}{2(n-1)} g\right),
$$

where Ric and Scal denote the Ricci and scalar curvatures of $g$. Set $g_{u}=e^{2 u} g_{0}$ for a metric pointwise conformal to the standard metric $g_{0}$ of $\mathbb{S}^{n}$ and $A_{u}$ for the Schouten tensor $A\left(g_{u}\right)$. Recall the transformation formula

$$
A_{u}=A_{0}-H_{0}(u)+d u \otimes d u-\frac{1}{2}|d u|_{0}^{2} g_{0},
$$

where $H_{u}$ and $|\cdot|_{u}$ stand for the Hessian and norm of the metric $g_{u}$. Let $\widetilde{A}_{u}$ be the symmetric endomorphism field defined by

$$
A_{u}(X, Z)=g_{u}\left(\widetilde{A}_{u} X, Z\right) \text { for all vector fields } Z,
$$

and define $\widetilde{H}_{u}$ likewise. Let $\lambda\left(A_{u}\right)$ be the $n$-tuple of eigenvalues of $\widetilde{A}_{u}$ (repeated according to their multiplicities); thus $\lambda\left(A_{0}\right)=\left(\frac{1}{2}, \ldots, \frac{1}{2}\right)$. Using (1), we get at $g_{u}$

$$
\frac{d}{d t}\left[\widetilde{A}_{(u+t v)}\right]_{t=0}=-\widetilde{H}_{u}(v)-2 v \widetilde{A}_{u} ;
$$

hence we have the following equation at $g_{0}$, which we record for later use:

$$
\frac{d}{d t}\left[\widetilde{A}_{t v}\right]_{t=0}=-\widetilde{H}_{0}(v)-v \mathrm{Id},
$$

where Id denotes the identity $\left(\begin{array}{l}1 \\ 1\end{array}\right)$-tensor. Finally, let $\mathfrak{f}$ be a $C^{\infty}$ symmetric real function defined in a domain $\mathfrak{D} \subset \mathbb{R}^{n}$ containing the $n$-tuple $\left(\frac{1}{2}, \ldots, \frac{1}{2}\right)$, satisfying $\mathfrak{f}\left(\frac{1}{2}, \ldots, \frac{1}{2}\right)=0$ and the nondegeneracy condition $d \mathfrak{f}\left(\frac{1}{2}, \ldots, \frac{1}{2}\right) \neq 0$. We have

$$
d \mathfrak{f}\left(\frac{1}{2}, \ldots, \frac{1}{2}\right)=(c, \ldots, c) \text { for some } c \in \mathbb{R}^{*} .
$$

Define the conformal f-scalar curvature operator $u \mapsto F(u)$ by

$$
F(u):=\mathfrak{f}\left[\lambda\left(A_{u}\right)\right] \text { for all } u \in C^{\infty}\left(\mathbb{S}^{n}\right) .
$$

In this context, the local $\mathfrak{f}$-Nirenberg problem is to characterize the local image of $F$ near $u=0$, namely

$$
\Im(F, 0)=\left\{F(u), u \in C^{\infty}\left(\mathbb{S}^{n}\right) \text { close to } 0\right\} .
$$

The difficulty arises from the fact, proved as property (i) in Section 3, that the linearization $L_{0}$ of $F$ at $u=0$ misses a vector space of dimension $n+1$, namely the eigenspace $\Lambda_{1}$ of first spherical harmonics; see [Berger et al. 1971], for example. 
Definition 1 [Delanoë 2003]. A local Fredholm resolution of $F$ at 0 is a couple of maps $(D, S)$ defined near 0 in $C^{\infty}\left(\mathbb{S}^{n}\right)$, where the map $D$ is a submersion valued in $\Lambda_{1}$ with $D(0)=0$, the map $S$ is valued in $C^{\infty}\left(\mathbb{S}^{n}\right)$ with $S(0)=0$, and the couple $(D, S)$ satisfies the identity

$$
F[S(f)]=f-D(f) .
$$

Definition 2 [Delanoë 2003]. A scalar constraint for $F$ at 0 is a real-valued submersion $K$ defined near 0 in $C^{\infty}\left(\mathbb{S}^{n}\right)$, such that $K \circ F \equiv 0$.

We will prove for $\mathfrak{I}(F, 0)$ the following results:

Theorem 1. There exists a local Fredholm resolution of $F$ at 0 . If $f$ is close enough to 0 in $C^{\infty}\left(\mathbb{S}^{n}\right)$ and invariant under a nontrivial group of isometries of $\left(\mathbb{S}^{n}, g_{0}\right)$ acting without fixed point, then $D(f)=0$; hence $f$ lies in $\mathfrak{I}(F, 0)$.

Theorem 2. There exists no scalar constraint for $F$ at 0 .

Identity (5) is a local nonlinear analogue of the Fredholm theorem; the second part of Theorem 1 is a local extension of existence results that are by now classical [Moser 1973; Escobar and Schoen 1986].

\section{Proofs}

The proofs of the theorems rely on two properties:

(i) The linearization $L_{0}$ of $F$ at $u=0$ is proportional to $\left(\Delta_{0}-n I\right)$.

(ii) For any $z \in \Lambda_{1}$, the coefficient of $(t z)^{3}$ in the expansion of $F(t z)$ at $t=0$ does not vanish.

Here $\Delta_{0}$ stands for the Laplacian of the standard metric $g_{0}$, whose first nonzero eigenvalue is equal to $n$, and $\Lambda_{1}$ is the corresponding $(n+1)$-dimensional eigenspace; see [Berger et al. 1971], for example. We require a standard lemma:

Lemma. Let $\mathfrak{D} \subset \mathbb{R}^{n}$ be a symmetric domain, $\varphi$ a symmetric $C^{1}$ real function defined on $\mathfrak{D}, \mathfrak{S}_{n}$ the open subset of real symmetric $n \times n$ matrices $a=\left(a_{i}^{j}\right)_{1 \leq i, j \leq n}$ whose $n$-tuple of eigenvalues $\lambda(a)$ lies in $\mathfrak{D}$. For $a \in \mathfrak{S}_{n}$, set $\Phi(a):=\varphi[\lambda(a)]$. Let $a_{0} \in \mathfrak{S}_{n}$ be diagonal, with diagonal entries $\left(\lambda_{01}, \ldots, \lambda_{0 n}\right)=: \lambda_{0}$. Then

$$
\frac{\partial \Phi}{\partial a_{i}^{j}}\left(a_{0}\right)=\delta_{i j} \frac{\partial \varphi}{\partial \lambda_{i}}\left(\lambda_{0}\right) .
$$

To verify property (i), set $\boldsymbol{f}(a):=\mathfrak{f}[\lambda(a)]$ and fix $v \in C^{\infty}\left(\mathbb{S}^{n}\right)$. Then

$$
L v=\left.\frac{d}{d t} F(t v)\right|_{t=0}=\sum_{i, j} \frac{\partial \boldsymbol{f}}{\partial a_{i}^{j}}\left(A_{0}\right) \frac{d}{d t}\left[\left(\widetilde{A}_{t v}\right)_{i}^{j}\right]_{t=0} .
$$


Applying the Lemma to $\varphi=\mathfrak{f}$ at $a_{0}=A_{0}$, we find

$$
L_{0} v=\sum_{i=1}^{n} \frac{\partial \mathfrak{f}}{\partial \lambda_{i}}\left(\frac{1}{2}, \ldots, \frac{1}{2}\right) \frac{d}{d t}\left[\left(\widetilde{A}_{t v}\right)_{i}^{i}\right]_{t=0} .
$$

Hence, from (3) and (4), we obtain $L_{0} v=c\left(\Delta_{0} v-n v\right)$, as required.

To check property (ii), using (3), we first observe the identity

$$
\frac{d}{d t}\left[\operatorname{Tr}\left(\widetilde{A}_{t v}\right)\right]_{t=0}=-\operatorname{Tr}\left(v \operatorname{Id}+\widetilde{H}_{0}(v)\right) \text { for all } v \in C^{\infty}\left(\mathbb{S}^{n}\right),
$$

where $\operatorname{Tr}$ stands for the trace. (The argument $v \operatorname{Id}+\widetilde{H}_{0}(v)$ on the right arises by applying the tilde construction of (2) to $v g_{0}+H_{0}(v)$, a Codazzi tensor [Ferus 1981] vanishing if and only if $v \in \Lambda_{1}$ [Obata 1962].) The preceding identity, applied to $v=z \in \Lambda_{1}$, simplifies greatly the calculation of $\left.\left(d^{3} / d t^{3}\right) F(t z)\right|_{t=0}$, which becomes equal to

$$
\sum_{i, j} \frac{\partial \boldsymbol{f}}{\partial a_{i}^{j}}\left(A_{0}\right) \frac{d^{3}}{d t^{3}}\left[\left(\widetilde{A}_{t v}\right)_{i}^{j}\right]_{t=0},
$$

or yet, by the Lemma and (4), to

$$
c \frac{d^{3}}{d t^{3}}\left[\operatorname{Tr}\left(\widetilde{A}_{t v}\right)\right]_{t=0} \equiv c \frac{d^{3}}{d t^{3}}\left[\sigma_{1}\left(\lambda\left(A_{t v}\right)\right)\right]_{t=0} .
$$

This brings us back to the scalar curvature case $\mathfrak{f}=\sigma_{1}-n / 2$ already treated in [Delanoë 2003, p. 36].

Proof of Theorem 1. Let $P_{1}$ be the $L^{2}\left(\mathbb{S}^{n}, g_{0}\right)$-orthogonal projection of $C^{\infty}\left(\mathbb{S}^{n}\right)$ onto $\Lambda_{1}$ [Berger et al. 1971]. The Fredholm theorem, combined with property (i), implies the existence of a unique solution $u \in C^{\infty}\left(\mathbb{S}^{n}\right)$ of the equation $L_{0} u=$ $f-P_{1} f$ with $P_{1} u=P_{1} f$; in other words, the equation $\left(L_{0}+P_{1}\right) u=f$ admits a unique solution in $C^{\infty}\left(\mathbb{S}^{n}\right)$. Hence, by the open mapping theorem, the map $\left(L_{0}+P_{1}\right)$ is an isomorphism of $C^{\infty}\left(\mathbb{S}^{n}\right)$. By an elliptic inverse function theorem argument, as in Theorem 7 of [Delanoë 2003], the map $u \mapsto F(u)+P_{1} u$ thus induces a diffeomorphism between neighborhoods of zero in $C^{\infty}\left(\mathbb{S}^{n}\right)$ : letting $S$ be its local inverse and setting $D=P_{1} \circ S$ we obtain (5), proving the first part of Theorem 1. The second part is proved as in Corollary 5 of the same work, namely: by naturality and uniqueness, the invariance of $f$ implies that of $S(f)$ and, since invariant functions are $L^{2}$ orthogonal to $\Lambda_{1}$ [Guan and Guan 2002], we indeed get $D(f)=P_{1}[S(f)]=0$.

Proof of Theorem 2. We argue by contradiction, as in [Delanoë 2003, pp. 36-37]. If $F$ admits a scalar constraint at 0 , by the naturality of $g \mapsto A(g)$ and the transitivity on $\mathbb{S}^{n}$ of the isometry group of $\left(\mathbb{S}^{n}, g_{0}\right)$, it must admit $n+1$ linearly independent scalar constraints at 0 [Delanoë 2003, Lemma 2]. If so, the map $D$ which occurs in the first part of Theorem 1 must satisfy $D \circ F \equiv 0$, by Theorem 2 of the same 
reference. But we readily see that this contradicts property (ii) above, by arguing as for Proposition 7 of that reference.

\section{Acknowledgments}

I thank Frédéric Robert for alerting me to the appearance of [Han 2006], and the referee for suggesting an improved presentation of Section 2.

\section{References}

[Berger et al. 1971] M. Berger, P. Gauduchon, and E. Mazet, Le spectre d'une variété riemannienne, Lecture Notes in Mathematics 194, Springer, Berlin, 1971. MR 43 \#8025 Zbl 0223.53034

[Delanoë 2003] P. Delanoë, "Local solvability of elliptic, and curvature, equations on compact manifolds", J. Reine Angew. Math. 558 (2003), 23-45. MR 2004e:53054 Zbl 1040.53047

[Delanoë and Robert 2007] P. Delanoë and F. Robert, "On the local Nirenberg problem for the $Q$ curvatures", Pacific J. Math. 231:2 (2007), 293-304.

[Escobar and Schoen 1986] J. F. Escobar and R. M. Schoen, "Conformal metrics with prescribed scalar curvature”, Invent. Math. 86:2 (1986), 243-254. MR 88b:58030 Zbl 0628.53041

[Ferus 1981] D. Ferus, "A remark on Codazzi tensors in constant curvature spaces", pp. 257 in Global differential geometry and global analysis (Berlin, 1979), edited by D. Ferus et al., Lecture Notes in Math. 838, Springer, Berlin, 1981. MR 83e:53045

[Guan and Guan 2002] B. Guan and P. Guan, "Convex hypersurfaces of prescribed curvatures", Ann. of Math. (2) 156:2 (2002), 655-673. MR 2003i:53046 Zbl 1025.53028

[Han 2006] Z.-C. Han, "A Kazdan-Warner type identity for the $\sigma_{k}$ curvature", C. R. Math. Acad. Sci. Paris 342:7 (2006), 475-478. MR 2006j:53045 Zbl 1099.53028

[Kazdan and Warner 1974] J. L. Kazdan and F. W. Warner, "Curvature functions for compact 2manifolds”, Ann. of Math. (2) 99 (1974), 14-47. MR 49 \#7949 Zbl 0273.53034

[Moser 1973] J. Moser, "On a nonlinear problem in differential geometry”, pp. 273-280 in Dynamical systems (Salvador, BA, 1971), edited by M. Peixoto, Academic Press, New York, 1973. MR 49 \#4018 Zbl 0275.53027

[Obata 1962] M. Obata, "Certain conditions for a Riemannian manifold to be iosometric with a sphere”, J. Math. Soc. Japan 14 (1962), 333-340. MR 25 \#5479 Zbl 0115.39302

[Sheng et al. 2007] W. M. Sheng, N. Trudinger, and X.-J. Wang, "The Yamabe problem for higher order curvatures”, J. Differential Geom. 77 (2007), 515-553. Zbl 05218126

[Stredder 1975] P. Stredder, "Natural differential operators on Riemannian manifolds and representations of the orthogonal and special orthogonal groups", J. Differential Geometry 10:4 (1975), 647-660. MR 54 \#3772 Zbl 0318.53046

[Viaclovsky 2000] J. A. Viaclovsky, "Some fully nonlinear equations in conformal geometry", pp. 425-433 in Differential equations and mathematical physics (Birmingham, AL, 1999), AMS/IP Stud. Adv. Math. 16, Amer. Math. Soc., Providence, RI, 2000. MR 2001i:53057 Zbl 01780483

Received February 27, 2007. Revised October 2, 2007. 
PHILIPPE DELANOË

UNIVERSité DE NiCE-SOPHIA ANTIPOLIS

LAboratoire J.-A. Dieudonné

PARC VALROSE

F-06108 NICE CEDEX 2

FRANCE

http://math.unice.fr/membres/delphi.html

Philippe.Delanoe@unice.fr 